\title{
GEOESTATÍSTICA MULTIVARIADA PARA A ESTIMATIVA DA TEMPERATURA MÉDIA ANUAL NO ESTADO DA BAHIA, BRASIL
}

\author{
Samira Luns Hatum de Almeida ${ }^{1}$ \\ Vinícius Agnolette Capelini ${ }^{2}$ \\ Jorge Tadeu Fim Rosas ${ }^{3}$ \\ Gabriel Dias de Oliveira ${ }^{4}$ \\ Julião Soares de Souza Lima ${ }^{5}$ \\ Samuel de Assis Silva ${ }^{6}$
}

\begin{abstract}
Resumo: O estudo da temperatura é muito importante para o desenvolvimento das culturas, sendo um estudo de grande aplicabilidade no cenário nacional e regional. O Estado da Bahia por apresentar o cultivo de diversas culturas em seu território, com destaque para o cacau, é uma região em que esse tipo de trabalho se torna necessário, uma vez que a temperatura não só está relacionada com o crescimento da lavoura, mas também à propagação e aparecimento de doenças. Com este trabalho, se objetivou estimar a temperatura média anual do Estado da Bahia por meio da geoestatística, a partir de sua relação com a altitude, utilizando o método de interpolação multivariada da cokrigagem. Com este método foi possível obter estimativas mais precisas, em que os resultados podem ser utilizados em diversas tomadas de decisão relacionada às culturas das áreas em questão e também no planejamento ambiental.

Palavras-chave: Cokrigagem; Krigagem; Interpolação multivariada; Mudanças climáticas globais.
\end{abstract}

\footnotetext{
${ }^{1}$ Agronomia/Universidade Federal do Espírito Santo, Brasil. E-mail: samiraluns@hotmail.com.

2 Agronomia/Universidade Federal do Espírito Santo, Brasil. E-mail: vinicius91ac@hotmail.com.

3 Agronomia/Universidade Federal do Espírito Santo, Brasil. E-mail: jorgetadeufimrosas@hotmail.com.

${ }^{4}$ Agronomia/Universidade Federal do Espírito Santo, Brasil. E-mail: gabriel.dias.oliveira@gmail.com.

${ }^{5}$ Agronomia/Universidade Federal do Espírito Santo, Brasil. E-mail: limajss@yahoo.com.br.

${ }^{6}$ Agronomia/Universidade Federal do Espírito Santo, Brasil. E-mail: samuel.assilva@gmail.com.
} 